\title{
Annexin A1 peptide is able to induce an anti-parasitic effect in human placental explants infected by Toxoplasma gondii
}

\author{
Marystela Fávero de Oliveira Cardoso ${ }^{\mathrm{a}, \mathrm{b}, 1}$, Jusciele Brogin Moreli ${ }^{\mathrm{a}, 1}$, Angélica Oliveira Gomes ${ }^{\mathrm{c}}$, \\ Caroline de Freitas Zanon $^{\mathrm{d}}$, Ana Elizabete Silva ${ }^{\mathrm{d}}$, Luana Ricci Paulesu ${ }^{\mathrm{e}}$, Francesca Ietta ${ }^{\mathrm{e}}$, \\ José Roberto Mineo ${ }^{\mathrm{f}}$, Eloísa Amália Ferro ${ }^{\mathrm{g}}$, Sonia Maria Oliani ${ }^{\mathrm{a}, \mathrm{d}, *}$ \\ ${ }^{a}$ Post-Graduation in Structural and Functional Biology, Federal University of São Paulo (UNIFESP), São Paulo, SP, Brazil \\ ${ }^{\mathrm{b}}$ Faceres School of Medicine, São José do Rio Preto, SP, Brazil \\ ${ }^{c}$ Department of Structural Biology, Institute of Biological and Natural Sciences, Federal University of Triângulo Mineiro (UFTM), Uberaba, MG, Brazil \\ d Department of Biology, Instituto de Biociências, Letras e Ciências Exatas; São Paulo State University (UNESP), São José do Rio Preto, SP, Brazil \\ e Department of Life Sciences, University of Siena, Siena, Italy \\ ${ }^{\mathrm{f}}$ Department of Immunology, Microbiology and Parasitology, Federal University of Uberlândia (UFU), Uberlândia, MG, Brazil \\ ${ }^{g}$ Department of Histology and Embryology, Federal University of Uberlândia (UFU), Uberlândia, MG, Brazil
}

\section{A R T I C L E I N F O}

\section{Keywords:}

Toxoplasmosis

Annexin A1

Placenta

$\mathrm{Ac}_{2-26}$ peptide

\begin{abstract}
A B S T R A C T
This study was conducted to investigate annexin A1 (ANXA1) functions in human placental explants infected with Toxoplasma gondii (T. gondii). We examined the first and third trimester placental explants infected with $T$. gondii ( $\mathrm{n}=7$ placentas/group) to identify the number and location of parasites, ANXA1 protein, potential involvement of formyl peptide receptors (FPR1 and FPR2), and COX-2 expressions by immunohistochemistry. Treatments with $\mathrm{Ac}_{2-26}$ mimetic peptide of ANXA1 were performed to verify the parasitism rate ( $\beta$-galactosidase assay), prostaglandin $\mathrm{E}_{2}$ levels (ELISA assay), and ANXA1, FPR1 and COX-2 expression in third trimester placentas. Placental explants of third trimester expressed less ANXA1 and were more permissive to T. gondii infection than first trimester placentas that expressed more ANXA1. $\mathrm{Ac}_{2-26}$ treatment increases endogenous ANXA1 and decreases parasitism rate, COX-2, and prostaglandin $\mathrm{E}_{2}$ levels. Altogether, these data provide further insight into the anti-parasitic and anti-inflammatory effects of ANXA1 in placentas infected with T. gondii.
\end{abstract}

\section{Introduction}

The intracellular protozoan Toxoplasma gondii (T. gondii) is an obligate intracellular apicomplexan parasite with a worldwide distribution that exerts a tremendous effect on human health [1]. In immunocompromised individuals this protozoan can cause severe consequences such as encephalitis, necrotic lesions in the central nervous system or retinochoroiditis [2,3]. Another important problem in public health is congenital infection. Toxoplasmosis is a pregnancy infection transmitted from mother to child and related to perinatal morbidity and mortality [4].

Some investigations suggest that the infectivity of $T$. gondii is justified in terms of a fetal-placental barrier, which operates regardless of a maternal immune response. In early stages of gestation, when the placental barrier is thicker, the transmission rate is smaller, and it increases as long as the barrier becomes progressively thinner with the advance of gestational age [5-7]. This barrier can be overcome by certain microorganisms such as the traditional ' $\mathrm{TORCH}$ ' pathogens ( $T$. gondii, other, rubella virus, cytomegalovirus and herpes simplex virus), resulting in potential fetal infection [8]. Some molecules derived from the placenta could be involved in this scenario [1,8]. Annexin A1 (ANXA1) is a calcium-dependent phospholipid binding protein that mediates glucocorticoid action, inhibits the prostaglandin synthesis, and limits cyclooxygenase 2 (COX-2) abundance. The biological effects of ANXA1 and its cleavage product $\mathrm{Ac}_{2-26}$ peptide are mediated by formyl peptide receptors (FPRs). In humans, three FPRs (FPR1, FPR2, and FPR3) regulate innate inflammatory responses [9-12].

In human placenta, ANXA1 location was first described on the syncytiotrophoblast cell surfaces [13,14]. Therefore, our group has investigated the expression of ANXA1 in deciduous, trophoblast, and stroma in partial and complete hydatidiform moles, showing its expression mainly in cytotrophoblast and syncytial knots. In addition,

\footnotetext{
* Corresponding author. IBILCE-UNESP, Department of Biology, Rua Cristovão Colombo, 2265, São José do Rio Preto, SP 15054-000, Brazil.

E-mail address: smoliani@ibilce.unesp.br (S.M. Oliani).

${ }^{1}$ M.F.O.C and J.B.M. contributed equally to this work.
} 
increased expression of this protein was shown in the cytotrophoblast of the hydropic villi in complete and partial hydatidiform mole [15]. We have also demonstrated an upregulation of endogenous ANXA1 in inflammatory cells of mice eyes and in the ARPE-19 cells (retinal pigmented epithelium) infected with $T$. gondii, suggesting a therapeutic target in the ocular toxoplasmosis [16].

Building upon the observations that the ANXA1 is produced by placenta and may interact on T. gondii transmission, we evaluated the potential protective effect of ANXA1 in first- and third-trimester human placental explants as a platform for innovative therapeutic strategies.

\section{Material and methods}

\subsection{Placenta samples and human chorionic villous explant cultures}

Seven samples of the third-trimester placentas (36-40 weeks of gestation) were collected after elective cesarean section deliveries, and seven first-trimester placentas (9-12 weeks of gestation) were obtained after elective termination of pregnancy. Samples from pregnant women with infections and positive serology for HIV and syphilis, multiple pregnancies, diabetes, fetal malformations, fetal death, and alcohol or illicit drugs habits were excluded. Placental tissues were washed in icecold sterile PBS (pH 7.2) and aseptically dissected using a microscope to remove endometrial tissue and fetal membranes up to $1 \mathrm{~h}$ after collection. Terminal chorionic villous containing five to seven free tips per explant was collected as described by De Oliveira Gomes et al. (2011) [5]. Explants were added to a 96-well plate (one per well) and cultured in $150 \mu \mathrm{L}$ RPMI 1640 (Invitrogen, Gibco, USA) medium supplemented with $10 \%$ fetal calf serum (Cultilab, Br) and antibiotics (Invitrogen, Carlsbad, USA) for $24 \mathrm{~h}$ at $37^{\circ} \mathrm{C}$ and $5 \% \mathrm{CO}_{2}$.

\subsection{Parasites}

Tachyzoites of T. gondii, 2F1 strain, which constitutively express cytoplasmic b-galactosidase and are derived from the RH strain, were a gift from Professor Vernon B. Carruthers, Medical School of Michigan University (Ann Arbor, MI, USA). These tachyzoites were propagated in BeWo cells maintained in RPMI 1640 medium (Invitrogen, Gibco, USA) supplemented with penicillin, streptomycin (Sigma-Aldrich Corp., St. Louis, MO, USA) and $2 \%$ fetal calf serum (Cultilab, Br) at $37^{\circ} \mathrm{C}$ and $5 \%$ $\mathrm{CO}_{2}$. The parasites were maintained by passages in this cell line for posterior infection of placental explants and for parasite intracellular proliferation assay, as previously described [17].

\subsection{Human chorionic villous explant infection and $A c_{2-26}$ treatment}

After $24 \mathrm{~h}$, explants were infected or not with the $2 \mathrm{~F} 1$ strain of $T$. gondii with $5 \times 10^{6}$ parasites/well [18] and evaluated after $48 \mathrm{~h}$ of infection. Alternatively, $24 \mathrm{~h}$ after infection, the samples were treated with 5 and $30 \mu \mathrm{M}$ of mimetic peptide $\mathrm{Ac}_{2-26}$ (Ac-AMVSEFLKQAWFIENEEQEYVQTVK; Thermo Fisher Scientifics, Waltham, MA, USA) dissolved in RPMI 1640 medium (Invitrogen, Gibco, USA) and incubated for an additional $24 \mathrm{~h}$. Then, villous explants were collected for morphologic, immunohistochemistry (ANXA1, FPR1, FPR2 and COX-2) and $\beta$-galactosidase analysis. Also, culture supernatants were collected and stored at $-80^{\circ} \mathrm{C}$ for measurement of human prostaglandin $\mathrm{E} 2\left(\mathrm{PGE}_{2}\right)$.

\subsection{Immunolocalization and quantification of T. gondii}

Samples of the infected villous explants of both first and third trimesters were fixed in $4 \%$ buffered formalin for $24 \mathrm{~h}$, dehydrated in graded ethanol and embedded in paraffin for immunolocalization and quantification of $T$. gondii. To rescue antigen, the sections were covered with trypsin solution $(0.05 \%$ trypsin and $0.1 \%$ calcium chloride) (Sigma-Aldrich Corp., St. Louis, MO, USA) for $30 \mathrm{~min}$ at $37^{\circ} \mathrm{C}$. Fragments were then incubated with acetic acid solution 5\% in TBS at room temperature to block endogenous phosphatase. Next, the sections were treated with normal goat serum in TBS $2.5 \%$ for blocking nonspecific sites and incubated with serum from Calomys callosus anti-T gondii (1:100). Fragments were incubated overnight at $4{ }^{\circ} \mathrm{C}$. To control the reaction, sections were incubated with normal serum from Calomys callosus. Samples were rinsed with TBS and incubated with goat antimouse IgG conjugated to biotin (Jackson Immuno Research, USA) for $1 \mathrm{~h}$ at $37^{\circ} \mathrm{C}$. Amplifications were done using $\mathrm{ABC}$ complex (complex streptavidin, biotin, alkaline phosphatase [ABC kit; Vector Laboratories, Inc., Burlington, CA, USA],) and developed with Fast-Rednaphthol (Sigma-Aldrich Corp., St. Louis, MO, USA) and counterstained with Mayer's hematoxylin. The quantification of tachyzoites was conducted on Axioskop 2-Mot Plus Microscope (Carl Zeiss, Jena, Germany), using AxioVision software. Ten random images of seven sections from each placenta (seven different placentas in each group) were used to count the number of tachyzoites.

\subsection{Immunohistochemical detection of ANXA1, COX-2, and the receptors FPR1 and FPR2 (FPRL1)}

Sections of placental explants $(3 \mu \mathrm{m})$ were incubated with sodium citrate buffer at $96^{\circ} \mathrm{C}$ for $30 \mathrm{~min}$. The endogenous peroxidase activity was blocked, and sections were incubated overnight at $4{ }^{\circ} \mathrm{C}$ with the primay rabbit polyclonal Abs anti-AnxA1 (1:2000) (Zymed Laboratories, Cambridge, UK), anti-FPR1 (1:1000) (Abcam, Cambridge, $\mathrm{UK}$ ), and anti-COX-2 (1:50) (Invitrogen, Paisley, UK) diluted in 1\% BSA. After washing, sections were incubated with a secondary biotinylated $\mathrm{Ab}$ (Dako, Cambridge, UK). Positive staining was detected using a peroxidase-conjugated streptavidin complex, and color was developed using DAB substrate (Dako, Cambridge, UK). Sections were counterstained with hematoxylin. Analysis was conducted on Axioskop 2-Mot Plus Microscope (Carl Zeiss, Jena, Germany), using AxioVision software for densitometric and quantitative analysis. In all, 140 random points of seven fields from each placenta (seven different placentas in each group) were used. Densitometric analysis was used to determine ANXA1, FPR1, and COX-2 intensity in trophoblast cells (40X) on an arbitrary scale from 0 to 255 .

\subsection{Detection of intracellular T. gondii by proliferation assay}

The third-trimester human placental explants (after the $\mathrm{Ac}_{2-26}$ treatment) were submitted to a colorimetric $\beta$-galactosidase assay to analyze the $T$. gondii intracellular proliferation. Villous explants were homogenized in radioimmunoprecipitation assay buffer (RIPA) [50 mmol/L Tris hydrochloride, $150 \mathrm{mmol} / \mathrm{L} \mathrm{NaCl}, 1 \%$ (v/v) Triton X$100,1 \%(\mathrm{w} / \mathrm{v})$ sodium deoxycholate, and $0.1 \%(\mathrm{w} / \mathrm{v})$ SDS; $\mathrm{pH} 7.5$ ] plus protease inhibitor cocktail tablets (Roche Diagnostics $\mathrm{GmbH}$, Mannheim, Germany). The homogenate was centrifuged at $15.000 \times \mathrm{g}$ for $15 \mathrm{~min}$ at $4{ }^{\circ} \mathrm{C}$, the supernatants were used for protein content measurement using the Bradford method. and $100 \mu \mathrm{g} / \mathrm{mL}$ of total protein was used to perform the colorimetric $\beta$-galactosidase assay. Then the total protein $(100 \mu \mathrm{g} / \mathrm{mL})$ was incubated with $160 \mu \mathrm{L}$ of assay buffer (100 mM phosphate buffer, $\mathrm{pH} 7.3,102 \mathrm{mM}$ mercaptoethanol and $9 \mathrm{mM} \mathrm{MgCl}_{2}$ ) and $40 \mu \mathrm{L}$ of $6.25 \mathrm{mM}$ CPRG (chlorophenol red- $\beta$-D-galactopyranoside; Roche, Indianapolis, IN, USA) for $30 \mathrm{~min}$. The enzymatic activity of $\beta$-galactosidase was measured at an absorbance of $570 \mathrm{~nm}$ using a kinetic plate reader (Titertek Multiskan Plus, Flow Laboratories, McLean, VA, USA).

\subsection{ELISA for $P G E_{2}$}

To detect $\mathrm{PGE}_{2}$ release in culture supernatants from explants of human placental villous, a compeptite immunoassay for the quantitative determination of $\mathrm{PGE}_{2}$ was performed according to the manufacturer's recommendations (Human Prostaglandin E2, Invitrogen, Paisley, UK). 


\subsection{Statistical analysis}

The statistics were performed using GraphPad software version 6.00. First, we performed the normality test Kolmogorov-Smirnov to determine if the data distribution was either parametric or nonparametric. Then differences between groups were evaluated by ANOVA, followed by either Bonferroni (parametric) or Dunn's test (non-parametric). The $t$-test was used to analyze the number of tachyzoites. A probability value less than 0.05 was considered significant.

\section{Ethics}

This cross-sectional study was approved by the local Research Ethics Committee from Federal University of São Paulo (CEP/UNIFESP: 47925115.1.1001.5505), Local Committee 2013 (University of Siena), and Federal University of Uberlândia (CEP/UFU: 006/09). Written informed consent was obtained from all subjects according to the principles of the Declaration of Helsinki.

\section{Results}

\subsection{Histopathology analysis}

The hematoxylin and eosin staining of sections was performed for structural integrity evaluation of chorionic villous in explants from first (Fig. 1A and B) and third trimester (Fig. 1C and D) of tissue infected (Fig. 1B and D) or not (Fig. 1A and C) with T. gondii. The syncytiotrophoblast, cytotrophoblast, stromal rich in fetal blood vessels and connective tissue were observed. In villous explants of the first trimester, the placental barrier was thicker due the presence of cytotrophoblast and syncytiotrophoblast (Fig. 1A). In addition, the tissue damage was less evident than that observed in third trimester placentas explants infected with $T$. gondii. In villous of third trimester, the syncytiotrophoblast was prevalent (Fig. 1C and D), and alterations such as detachment and disorganization of syncytiotrophoblast (Fig. 1B and D), fetal connective tissue destruction of villous stroma (Fig. 1D) and nuclei fragmentation (Fig. 1B and D) were more evident in T. gondii infection than in control placentas.

\subsection{Gestational age of placenta modulates the distribution and number of T. gondii}

To compare the number and localization of $T$. gondii in placental villous of first and third trimesters, the immunohistochemistry with anti- $T$ gondii antibody was performed in infected samples. Interestingly, different locations and numbers of parasite were observed in placenta explants culture of the first and third trimesters. In first trimester explants, parasites were observed only in trophoblast cells (Fig. 1E) and in lower numbers (Fig. 1G). Conversely, in third trimester explants, parasites were found also in stroma (Fig. $1 \mathrm{~F}$ ) and in higher numbers (Fig. 1G).

\subsection{ANXA1, FPR1 and COX-2 are differentially expressed in T. gondii infected placental cells from the first and third trimesters of gestation}

The relevance of COX-2, ANXA1, and their receptors (FPR1 and FPR2) in T. gondii conditions was investigated through densitometric analysis of immunohistochemistry reactions in placental tissues. In general, the ANXA1 localization (Fig. 2A and B) was coincident with the FPR1 receptor (Fig. 2E and F) in trophoblast cells of the first trimester. In third trimester explants, the immunoreactivity for ANXA1 (Fig. 2C and D) and FPR1 (Fig. 2G and H) were observed in syncytiotrophoblast and mesenchymal cells in both control and infected villous. There was no immunoreactivity for FPR2 receptor (Fig. 2I) in explants from the first and third trimester of either control or infected groups.

The densitometric analysis of ANXA1 (Fig. 2K) and FPR1 (Fig. 2L) considering trophoblastic cells of first trimester placental explants showed high immunoreactivity of ANXA1 in cytotrophoblast compared to the syncytiotrophoblast in the control group. After T. gondii infection, ANXA1 expression decreased in cytotrophoblast and FPR1 expression increases only in syncytiotrophoblast. When the analyses were performed considering positive cells types from trophoblast and stromal region (Fig. 2M and $\mathrm{N}$ ), the immunoreactivity for ANXA1 decreased in control group in explants of the third trimester compared with the control group of first trimester explants (Fig. 2M). The FPR1 expression (Fig. $2 \mathrm{~N}$ ) increased in all groups compared with the control group of the first trimester.

Furthermore, the immunostaining of COX-2 (Fig. s1) was observed in cells located in the mesenchymal in explants of the first trimester (Figures S1 A and B) with or without infection and preferably in the syncytiotrophoblast in explants of the third trimester (Figures S1 C and D). COX-2 expression in explants of the third trimester was reduced in T. gondii infection compared with control (Figure S1 E).

\section{4. $A c_{2-26}$ peptide treatment can reduce parasitism rate in placental explants of the third trimester of pregnancy}

After observing that third trimester placentas presented low expression of ANXA1 and higher numbers of parasites than first trimester placentas, the culture explants of third trimester placentas were treated with $5 \mu \mathrm{M}$ and $30 \mu \mathrm{M}$ of $\mathrm{Ac}_{2-26}$ mimetic peptide of ANXA1. Then the explants were submitted to a colorimetric $\beta$-galactosidase assay to analyze the $T$. gondii intracellular proliferation. Interestingly, intracellular proliferation of $T$. gondii was higher in the untreated group and lower in samples treated with $5 \mu \mathrm{M}$ or $30 \mu \mathrm{M}$ of $\mathrm{Ac}_{2-26}$ peptide (Fig. 3).

4.5. ANXA1 expression is modulated by $A c_{2-26}$ peptide treatment in $T$. gondii infected placental cells from the third trimester

The densitometric analysis of immunohistochemistry reactions were performed to verify if the $\mathrm{Ac}_{2-26}$ treatment is able to modulate endogenous ANXA1 and their receptor FPR1 mainly in placentas with reduction of $T$. gondii proliferation. The ANXA1 and FPR1 were observed mainly in syncytiotrophoblast cells of all groups (Figs. 4 and 5). In general, the $\mathrm{Ac}_{2-26}$ treatment was able to modulate the endogenous ANXA1 in control and T. gondii groups such as a reduction of ANXA1 expression in control group treated with $5 \mu \mathrm{M}$. Still, in the groups treated with $5 \mu \mathrm{M}$, ANXA1 expression increased in T. gondii group compared with the control group in the same condition (Fig. 4G).

Moreover, there was an increase of FPR1 expression in the infected group compared with the control group, both in the untreated condition (Fig. 5G). Thus, the infection by T. gondii modulates the FPR1 receptor (independent of treatment), and the $\mathrm{Ac}_{2-26}$ treatment abrogates this effect.

4.6. Infection with $T$. gondii and $A c_{2-26}$ treatment can reduce $C O X-2$ and PGE2 levels in placental explants of the third trimester of pregnancy

ANXA1 is a protein that displays potent anti-inflammatory properties by inhibiting the prostaglandin synthesis and limiting COX-2 [10]. The relevance of these molecules in placental explants infected with $T$. gondii and treated with $\mathrm{Ac}_{2-26}$ mimetic peptide of ANXA1 was investigated through the immunolocalization of COX-2 and measuring PGE2 levels by ELISA. Both COX-2 and PGE2 were detectable in the placentas of all groups (Fig. 6A-F). Densitometric analysis of COX-2 immunohistochemical (Fig. 6G) and PGE2 levels (Fig. 6H) demonstrated reduction of these molecules in all groups compared with the control group without treatment. 


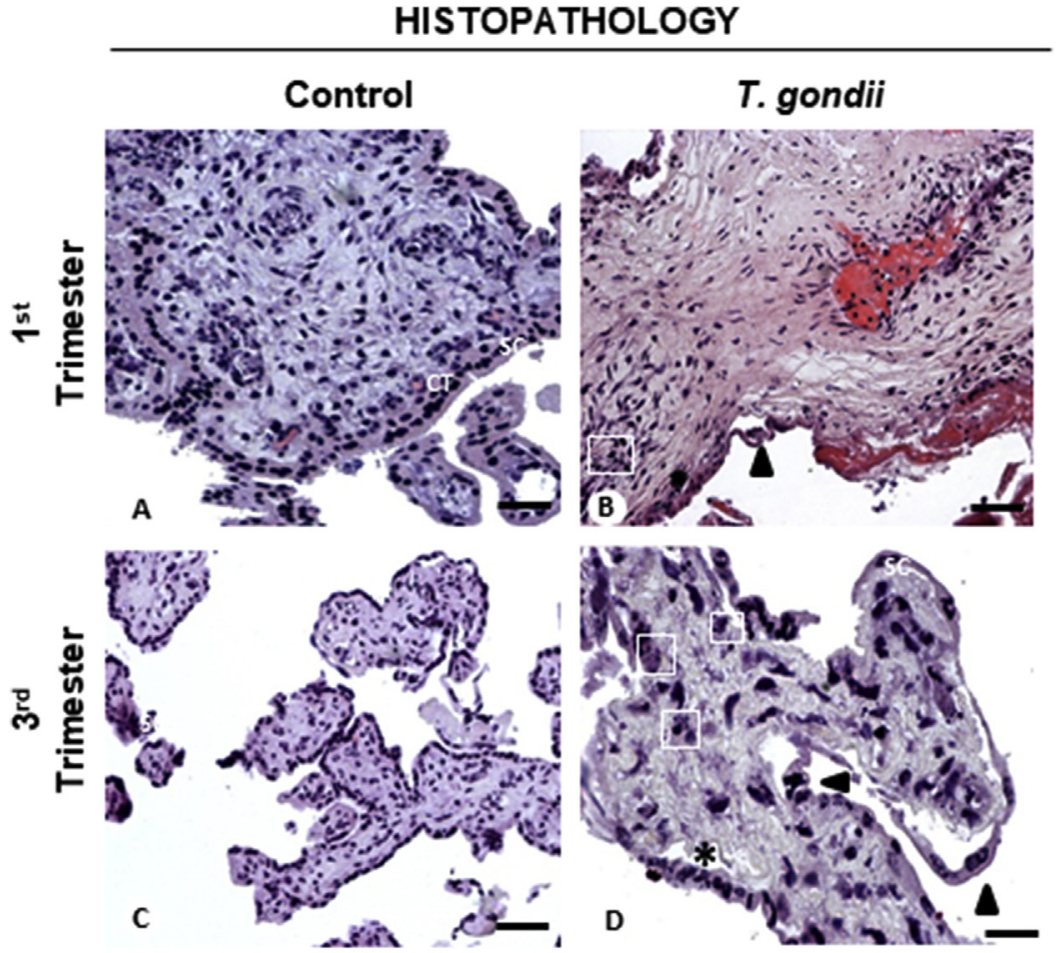

IMMUNOLOCALIZATION AND QUANTIFICATION OF $T$. gondii
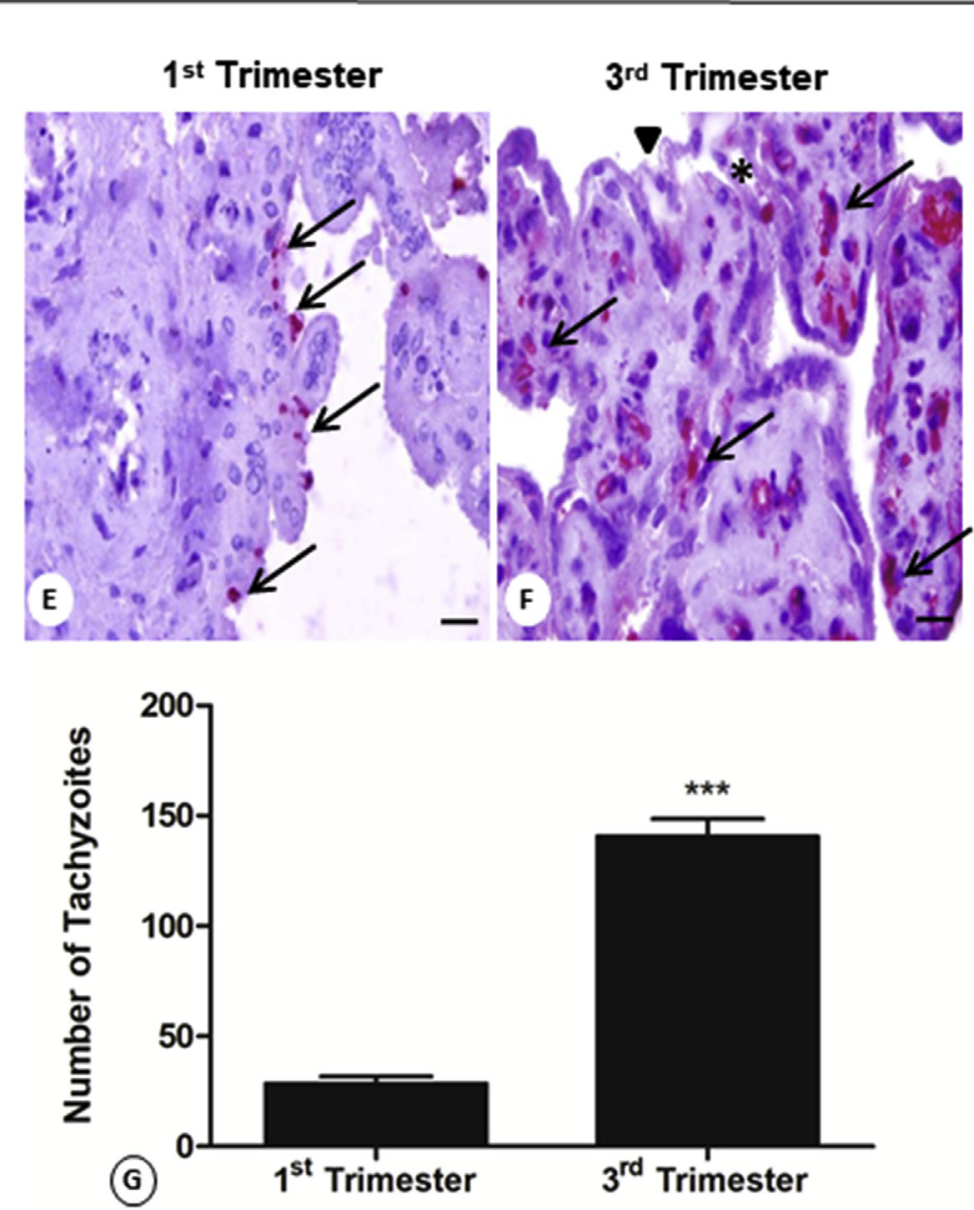

Fig. 1. Histopathology and parasite analysis in placental explants infected with Toxoplasma gondii. Villous explants showing syncytiotrophoblast (SC), cytotrophoblast (CT) and stroma cells. Detachment and disorganization of the SC (arrowhead), fetal connective tissue destruction of the villous stroma (asterisk), and nuclei fragmentation (square) were more evident in T. gondii-infected explants [B, $\mathrm{D}, \mathrm{E}, \mathrm{F}]$ compared to control explants $[\mathrm{A}, \mathrm{C}]$, especially in third trimester placentas [D, F]. Hematoxylin-eosin stain [AD]. Immunolocalization (arrows) [E, F] and quantification [G].of T. gondii. Counterstaining: Mayer hematoxylin [E, F]. Sections: $3 \mu \mathrm{m}$. Bars: $10 \mu \mathrm{m}$. Values as mean \pm S.D., $* * * p<0.001$ versus first trimester ( $\mathrm{n}=7$ samples/group). 

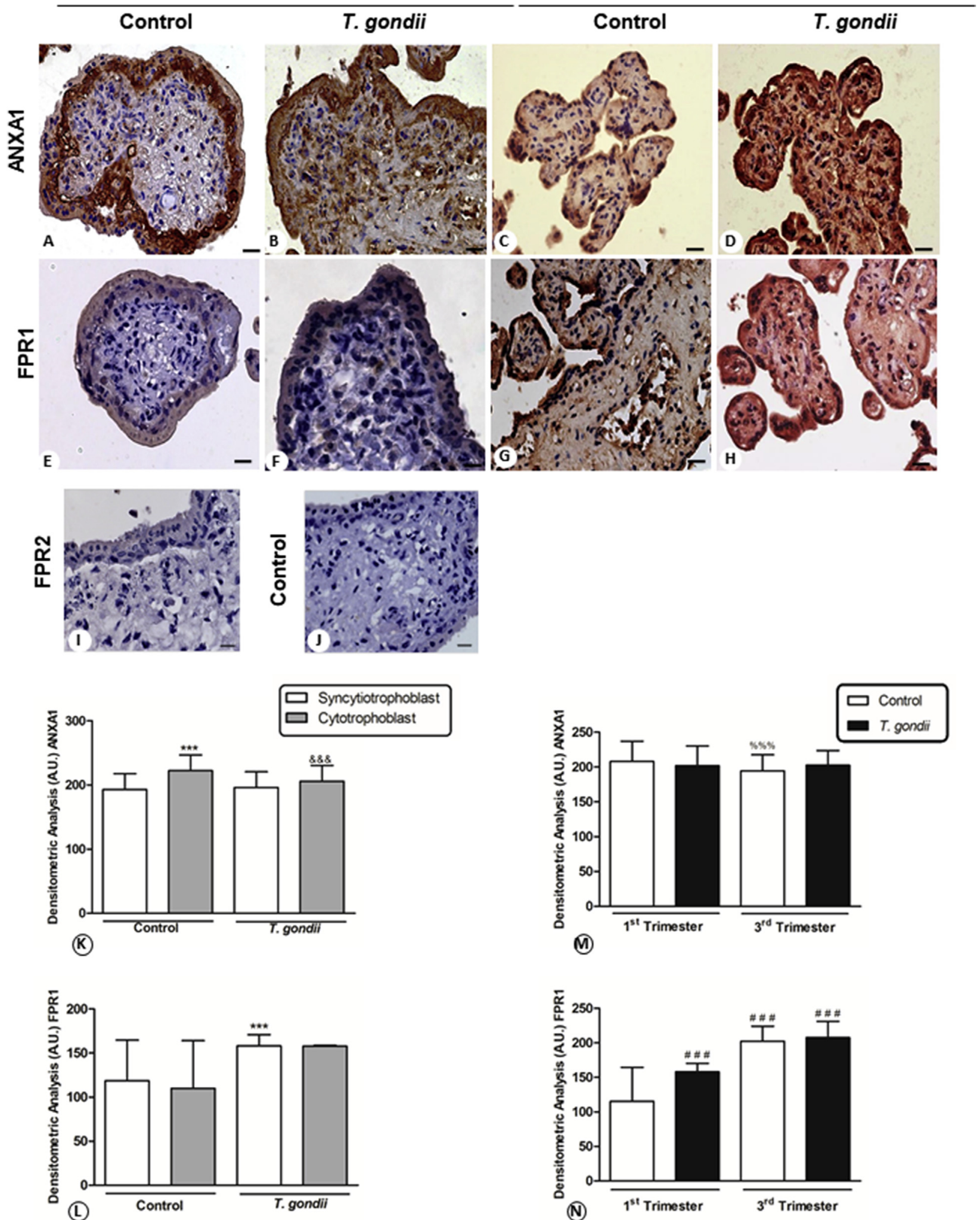

Fig. 2. Influence of Toxoplasma gondii infection on ANXA1 and FPR1 expression in placental explants of the first and third trimesters of pregnancy. Immunohistochemical [A-J] and quantitative analysis of ANXA1 [K, M] and FPR1

$[\mathrm{L}, \mathrm{N}]$. The analyses in $[\mathrm{K}]$ and $[\mathrm{L}]$ were performed only in first trimester placentas of control and infected groups separately in syncytiotrophoblast and cytotrophoblast cells. In M and N, all ANXA1 and FPR1 positive cells (without syncytiotrophoblast and cytotrophoblast differentiation) were considerate of quantification in both first and third trimester placentas. Absence of immunostaining in the FPR2 receptor [I] and control of reaction [J]. Counterstaining: hematoxylin. Sections: $3 \mu \mathrm{m}$. Bars: $10 \mu \mathrm{m}$. Values as mean \pm S.D.; $* * * p<0.001$ versus control syncytiotrophoblast in $\mathrm{K}$ and $\mathrm{L} ; \& \& \& p<0.001$ versus control cytotrophoblast in $\mathrm{K} ; \% \% \%$ $p<0.001$ versus first trimester control in $[\mathrm{M}] ; \# \# \# p<0.001$ versus first trimester control in $\mathrm{N}$; ( $=7$ samples/group). 


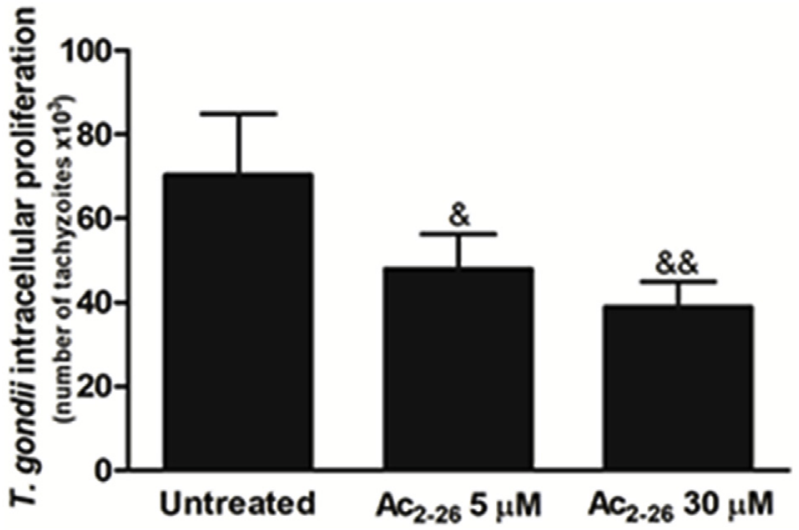

Fig. 3. Effect of $\mathrm{Ac}_{2-26}$ peptide treatments on parasitism rate in third trimester placental explants. Parasitism rate measured by a colorimetric $\beta$-galactosidase assay for showing reduced parasite proliferation in the explants treated with peptide in the concentrations of $5 \mu \mathrm{M}$ and $30 \mu \mathrm{M}$. Values as mean \pm S.D. $\& p<0.05$ and $\& \& p<0.01$ versus untreated ( $\mathrm{n}=7$ samples/ group).

\section{Discussion}

The trophoblast cells are an important component of the placental barrier, and they are involved in the protective mechanism to prevent vertical transmission of parasites, especially $T$. gondii $[6,8,19]$. When the parasite overcomes the placental barrier, the health of both mother and offspring is compromised [6].

Previous reports of our group have shown that ANXA1 may be one important therapeutic target in the ocular toxoplasmosis [16]. Here, we identify that placental explants of the third trimester expressed less ANXA1 and were more permissive to $T$. gondii infection than placentas of the first trimester that expressed more ANXA1. To confirm the relationship between ANXA1 and T. gondii infection, we conduct a culture of third trimester explants with $\mathrm{Ac}_{2-26}$ treatment and observed parasite reduction after treatment.

The biological changes of the placenta through gestation and the lower ANXA1 expression in third trimester placentas justify our different findings related to distribution and number of $T$. gondii in placenta. In the early stages of gestation, the placental barrier is thicker; the transmission rate is lower and increases this transmission when the barrier becomes progressively thinner with the advance of gestational age $[5,7,19]$. In fact, in first trimester placentas, we observed higher expression of ANXA1 and lower number of parasites, which were present only in trophoblast cells. Differently, third trimester placentas
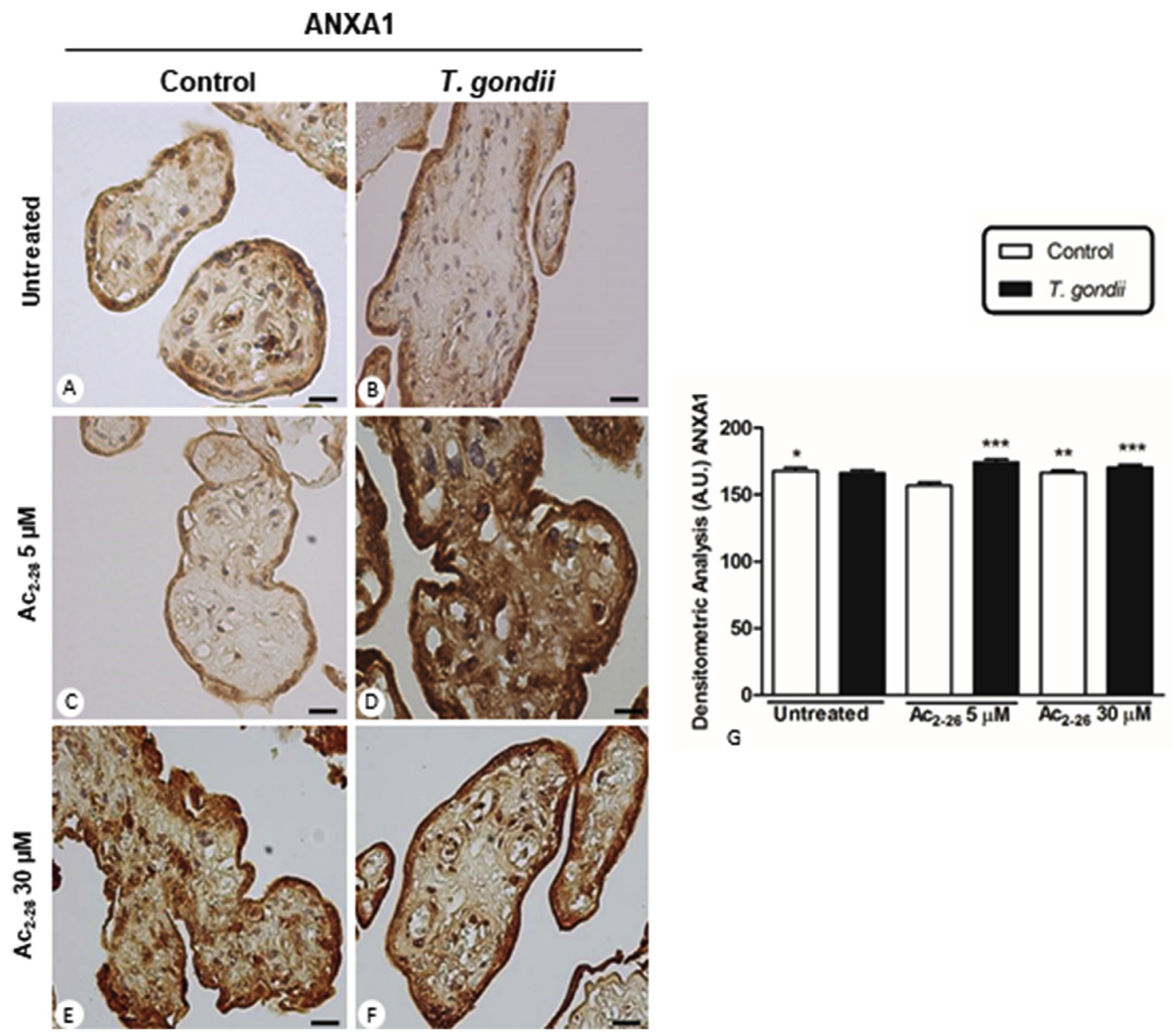

Fig. 4. Effect of $\mathrm{Ac}_{2-26}$ peptide treatments on endogenous ANXA1 expression in third trimester placental explants. Immunohistochemical for ANXA1 in the villous explants untreated [A, B] or treated with $5 \mu \mathrm{M}[\mathrm{C}, \mathrm{D}]$ and $30 \mu \mathrm{M}[\mathrm{E}, \mathrm{F}]$ of $\mathrm{A}_{\mathrm{c} 2-26}$ peptide. Quantitative analysis of ANXA1 expression [G]. Counterstaining: hematoxylin. Sections: $3 \mu \mathrm{m}$. Bars: $10 \mu \mathrm{m}$. Values as mean \pm S.D. ${ }^{*} p<0.05,{ }^{* *} p<0.01$ and ${ }^{* * *} p<0.001$ are versus control group treated with $5 \mu \mathrm{M}$ in [G]. ( $\mathrm{n}=7$ samples/group). 


\section{FPR1}
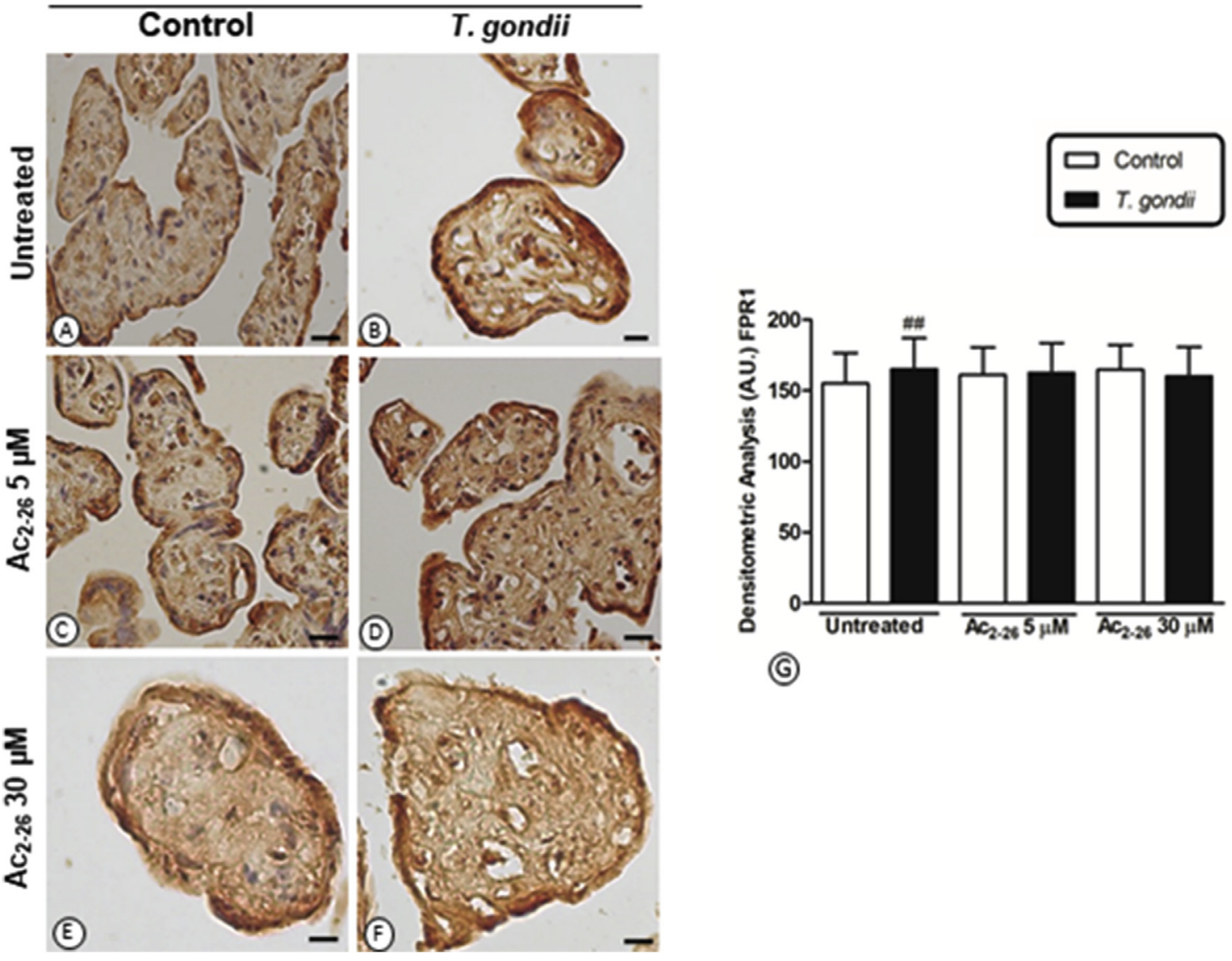

(G)

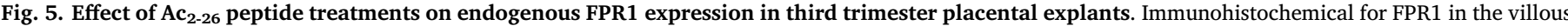

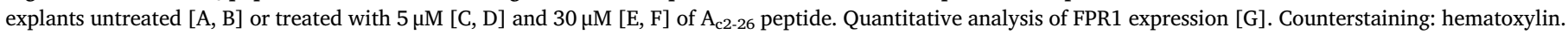
Sections: $3 \mu \mathrm{m}$. Bars: $10 \mu \mathrm{m}$. Values as mean \pm S.D. \#\#p $<0.01$ versus control group untreated in [G] (n $=7$ samples/group).

presented parasites in trophoblast cells and in stroma, with increased numbers of parasites and decreased ANXA1 expression. Another important change was the higher ANXA1 expression in first trimester placentas is due to cytotrophoblast presence, since we observed more ANXA1 expression in cytotrophoblast than syncytiotrophoblast. A previous study also identified differential expression of ANXA1 in placenta and amnion, where this protein may contribute to increased phospholipase A2 activity, arachidonic acid mobilization, and prostaglandin production during labor in humans [20].

In addition to biological changes in the placental barrier through gestation $[5,7,19]$ and the ANXA1 modulation proposed here, the literature demonstrated other routes involved in vertical transmission of pathogens. This includes infection of endothelial cells in the maternal microvasculature and spreading to invasive extravillous trophoblasts; trafficking of infected maternal immune cells across the placental barrier; paracellular or transcellular transport from maternal blood across the villous trees and into the fetal capillaries; and damage to the villous tree and breaks in the syncytiotrophoblast layer [8]. In our study model, severe tissue damage such as detachment and disorganization of syncytiotrophoblast observed in the placental barrier (specially in third trimester explants) infected with T. gondii might explain at least partially the infection and $T$. gondii transmission in these placentas.

Treatment of cell explants from the third trimester with $\mathrm{Ac}_{2-26}$ peptide $(5$ and $30 \mu \mathrm{M})$ showed an increase of endogenous ANXA1 expression in syncytiotrophoblast of $T$. gondii group and a decrease of parasitism, which also indicates a possible role of ANXA1 as an antiinfective agent. Previous results of our group also showed that infection by $T$. gondii modulates positively ANXA1 expression in retinal pigment of epithelium involved in inflammatory response [16]. In the context of toxoplasmosis, balance between inflammatory and anti-inflammatory responses is crucial, since inflammation in excess could result in deleterious tissue damage [21]. ANXA1 is an important and well characterized protein involved in resolution of inflammation in promoting tissue repair and restoring homeostasis [22,23].

Externalization and secretion of ANXA1 occurs through interaction with the peptide formyl receptors (FPRs) [9,24]. Classically, the FPRs are involved in recruitment and activation of leukocytes and promotion of cell motility $[9,24]$. ANXA1 protein activates the cascade of mitosis using cyclin D1 and FPR1/2 [25]. In fact, some studies using pancreatic and adrenal epithelial cells indicate the proliferative role of endogenous ANXA1 [26,27]. Our previous findings also related ANXA1 with proliferation, differentiation, and apoptosis in gestational trophoblastic disease [15] and endometriosis through interaction with FPR1 receptor [28].

Classically, FPRs mediate ANXA1 signaling to promote resolution of inflammation. FPR1 mediates the actions of ANXA1 and its peptide Ac2-26 in nonimmune cells, while FPR2/ALX seems predominant on myeloid cells [29-31]. The pro-repair properties of endogenous ANXA1 and its peptide were described as dependent of both FPR1 and FPR2 in epithelial wound repair [30] and in the suppressive effect of peptide Ac2-26 on fibrotic responses induced by silica in lungs [31]. In contrast, the immunoreactivity for FPR2 in trophoblast cells was not detectable in our placental samples, although the expression of FPR1 was coincident with the regions characterized for ANXA1 but with different patterns of expression. The possible interaction between endogenous ANXA1 and FPR1 observed in our findings supports the idea of one 

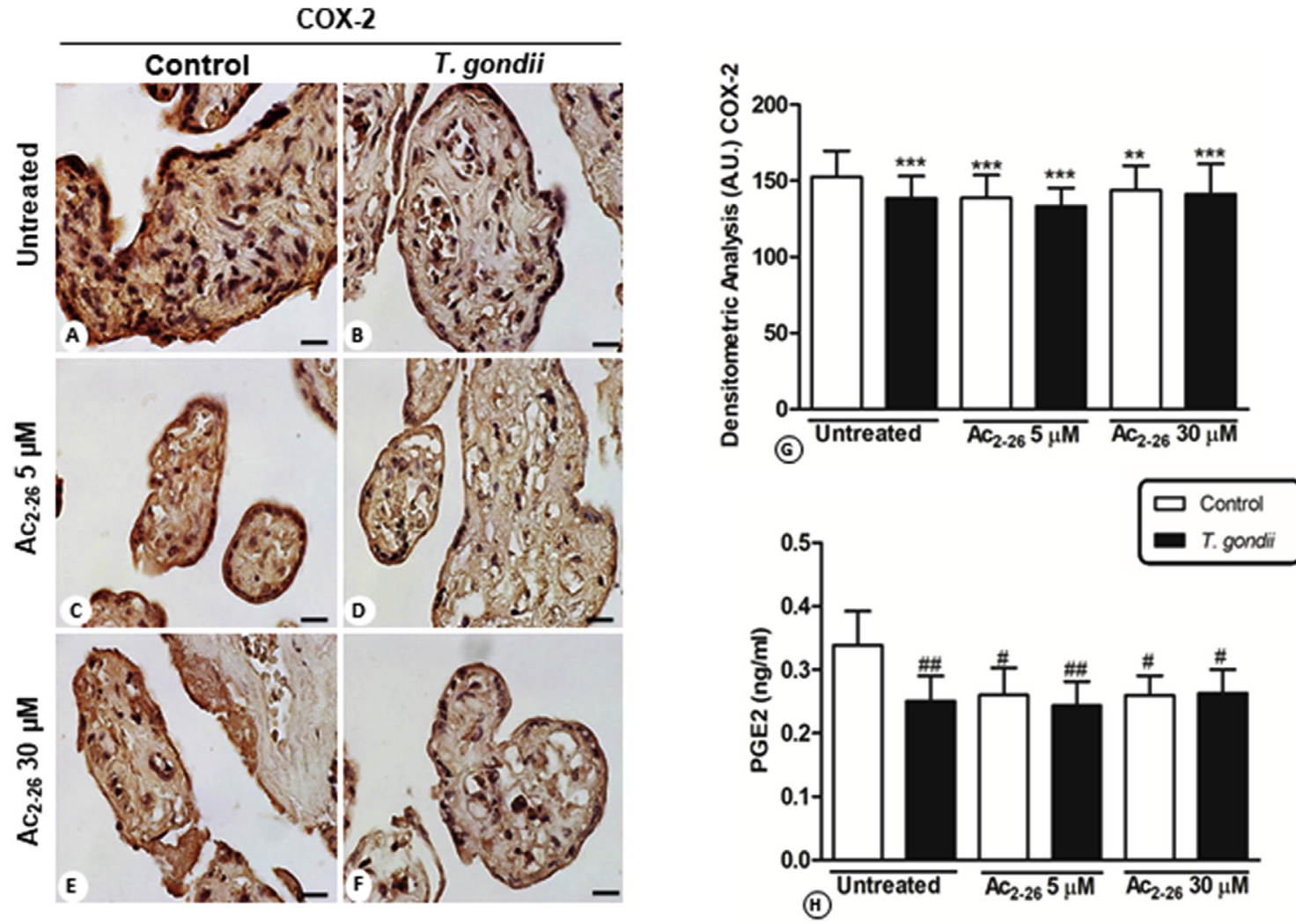

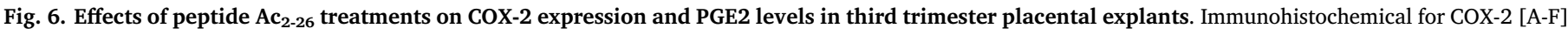

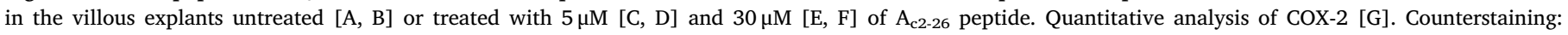

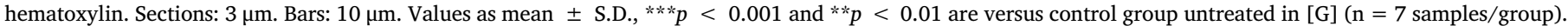

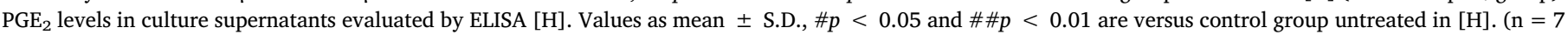
samples/group).

mechanism being involved in ANXA1 action through this receptor, especially in the placenta. Previous studies also showed ANXA1/FPR1 interaction in both leukocytes and undifferentiated glandular pattern of endometriotic lesions [12,28,32,33].

Recent investigations using human retinal pigment epithelium cells (ARPE-19) activated by LPS showed ANXA1 and FPR expression as well as the anti-inflammatory effect of peptide $\mathrm{Ac}_{2-2}$ by reduction of inflammatory mediators such as IL-6, IL-8, and COX-2 [34]. Molecular analysis of NF- $\mathrm{KB}$ translocation and gene expression of those mediators indicated that the protective effects of ANXA1 occur independently of the NF- $\mathrm{KB}$ signaling pathway and possibly in a post-transcriptional manner [34]. In our model, the anti-inflammatory effects of the $\mathrm{Ac}_{2-26}$ peptide were demonstrated by decreased COX-2 expression and $\mathrm{PGE}_{2}$ levels in placentas treated with $\mathrm{Ac}_{2-26}$. It is known that $\mathrm{COX}-2$ promotes arachidonic acid breakdown in the plasma membrane, leading to synthesis of prostaglandins and leukotrienes such as prostaglandin E2 [35], justifying our findings. In addition, ANXA1 inhibited COX-2 expression in macula densa through activation of FPR1 in kidney [36].

Independent of the treatment, COX-2 and $\mathrm{PGE}_{2}$ levels were lower in the infected group when compared to the control group. $\mathrm{PGE}_{2}$ is known to be important for the persistence of $T$. gondii in Bewo cells [37]. $\mathrm{PGE}_{2}$ is associated with immunosuppression, which helps parasites avoid the killing effect of the immune system in an immune competent host [37]. Immunosuppressive effects of $\mathrm{PGE}_{2}$ were observed in human trophoblast cells infected with $T$. gondii, demonstrating a critical mediator that contributed to parasite proliferation.

\section{Conclusions}

This investigation has gained insight into the protective effect of endogenous ANXA1 in human placentas infected with $T$. gondii. In addition, the treatment with the mimetic peptide of ANXA1, Ac ${ }_{2-26}$, can induce anti-parasitic and anti-inflammatory effects in placental villous explants of the third trimester. These observations shed light on the mechanisms regulating the functions of this important protein in the placental biology.

\section{Author contributions}

M.F.O.C., A.O.G., J.B.M., A.E.S. and C.F.Z. performed experiments and analysed the data obtained. M.F.O.C., A.O.G., J.B.M., E.A.F. and S.M.O. contributed to the writing of the manuscript. E.A.F., L.R.P., F.I. and J.R.M. contributed with placental samples and experiments. S.M.O. was the leader of the project, and contributed to the writing of the manuscript. All authors read and approved the final manuscript.

\section{Conflicts of interest}

The authors declare that they have no conflict of interest.

\section{Acknowledgment}

This work was supported by the Fundação de Amparo à Pesquisa do Estado de São Paulo - FAPESP [grants 2013/08273-6 to M.F.O.C and 2016/02012-4 to S.M.O.]; Conselho Nacional de Desenvolvimento Científico e Tecnológico - CNPq [grant 308144/2014-7 to S.M.O and 303252/2015-4 to E.A.F]. The authors would like to thank Dr. Nathália Maciel Maniezzo Stuchi and Rafaela Batista Molás for technical support. 


\section{Appendix A. Supplementary data}

Supplementary data related to this article can be found at https:// doi.org/10.1016/j.micpath.2018.07.005.

\section{References}

[1] C. Yan, L.J. Liang, K.Y. Zheng, X.Q. Zhu, Impact of environmental factors on the emergence, transmission and distribution of Toxoplasma gondii, Parasit. Vectors (2016), https://doi.org/10.1186/s13071-016-1432-6.

[2] M.S. Ferreira, A.S. Borges, Some aspects of protozoan infections in immunocompromised patients: a review, Mem. Inst. Oswaldo. Cruz 97 (2002) 443-457.

[3] J.P. Dubey, E.G. Lago, S.M. Gennari, C. Su, J.L. Jones, Toxoplasmosis in humans and animals in Brazil: high prevalence, high burden of disease, and epidemiology, Parasitology 139 (2012) 1375-1424.

[4] H.S. Oz, Maternal and congenital toxoplasmosis, currently available and novel therapies in horizon, Front. Microbiol. (2014), https://doi.org/10.3389/fmicb. 2014.00385.

[5] A. De Oliveira Gomes, D.A. de Oliveira Silva, N.M. Silva, B. de Freitas Barbosa, P.S. Franco, M.B. Angeloni, et al., Effect of macrophage migration inhibitory factor (MIF) in human placental explants infected with Toxoplasma gondii depends on gestational age, Am. J. Pathol 178 (2011) 2792-2801.

[6] J.R. Robbins, A.I. Bakardjiev, Pathogens and the placental fortress, Curr. Opin. Microbiol. 15 (2012) 36-43.

[7] N. Arora, Y. Sadovsky, T.S. Dermody, C.B. Coyne, Microbial vertical transmission during human pregnancy, Cell. Host. Microbe 21 (2017) 561-567.

[8] C.B. Coyne, H.M. Lazear, Zika virus - reigniting the TORCH, Nat. Rev. Microbiol. 14 (2016) 707-715.

[9] Y. Le, P.M. Murphy, J.M. Wang, Formyl-peptide receptors revisited, Trends. Immunol 23 (2002) 541-548.

[10] M. Perretti, The annexin 1 receptor(s): is the plot unravelling? Trends. Pharmacol. Sci. 24 (2003) 574-579.

[11] F. D'Acquisto, M. Perretti, R.J. Flower, Annexin-A1: a pivotal regulator of the innate and adaptive immune systems, Br. J. Pharmacol. 155 (2008) 152-169.

[12] T.S. Gastardelo, A.S. Damazo, J. Dalli, R.J. Flower, M. Perretti, S.M. Oliani, Functional and ultrastructural analysis of annexin A1 and its receptor in extravasating neutrophils during acute inflammation, Am. J. Pathol 174 (2009) 177-183.

[13] K.G. Moore, F. Goulet, A.C. Sartorelli, Purification of annexin I and annexin II from human placental membranes by high-performance liquid chromatography protein, Expr. Purif 3 (1992) 1-7.

[14] M. Sun, Y. Liu, W. Gibb, Distribution of annexin I and II in term human fetal membranes, decidua and placenta, Placenta 17 (1996) 181-184.

[15] M.F.O. Cardoso, A.P. Girol, E.A.V. Ferro, S.M. Oliani, Annexin A1 and formyl peptide receptor in gestational trophoblastic diseases: focus on proliferation and apoptosis, Journal of International Research in Medical and Pharmaceutical Sciences 4 (2015) 40-46.

[16] K.K. Mimura, R.C. Tedesco, K.S. Calabrese, C.D. Gil, S.M. Oliani, The involvement of nti-inflammatory protein, annexin A1, in ocular toxoplasmosis, Mol. Vis 18 (2012) 1583-1593.

[17] A.W. Pfaff, O. Villard, J.P. Klein, M. Mousli, E. Candolfi, Regulation of Toxoplasma gondii multiplication in BeWo trophoblast cells: cross-regulation of nitric oxide production and polyamine biosynthesis, Int. J. Parasitol 35 (2005) 1569-1576.

[18] A.S. Castro, C.M.O.S. Alves, M.B. Angeloni, A.O. Gomes, B.F. Barbosa, P.S. Franco, et al., Trophoblast cells are able to regulate monocyte activity to control Toxoplasma gondii infection, Placenta 34 (2013) 240-247.

[19] J.R. Robbins, V.B. Zeldovich, A. Poukchanski, J.C. Boothroyd, A.I. Bakardjiev, Tissue barriers of the human placenta to infection with Toxoplasma gondii, Infect. Immun 80 (2012) 418-428.

[20] L. Myatt, J. Hirth, W.V. Everson, Changes in annexin (lipocortin) content in human amnion and chorion at parturition, J. Cell. Biochem 50 (1992) 363-373.

[21] J. Iqbal, M. Al-Awadhi, Toxoplasmosis: role of cytokines in disease modulation \& tissue pathology, Ann. Clin. Pathol 4 (2016) 2792-2801.

[22] G. Leoni, A. Nusrat, Annexin A1: shifting the balance towards resolution and repair, Biol. Chem. 397 (2016) 971-979.

[23] T. Gobbetti, S.N. Cooray, Annexin A1 and resolution of inflammation: tissue repairing properties and signalling signature, Biol. Chem. 397 (2016) 981-993.

[24] F.N. Gavins, M.J. Hickey, Annexin A1 and the regulation of innate and adaptive immunity, Front. Immunol 27 (2012) 1-11.

[25] T. Khau, S.Y. Langenbach, M. Schuliga, T. Harris, C.N. Johnstone, R.L.S. Anderson, et al., Annexin-1 signals mitogen-stimulated breast tumor cell proliferation by activation of the formyl peptide receptors (FPRs) 1 and 2, FASEB. J. 25 (2011) 483-496.

[26] X.F. Bai, X.G. Ni, P. Zhao, S.M. Liu, H.X. Wang, B. Guo, et al., Overexpression of annexin 1 in pancreatic cancer and its clinical significance, World. J. Gastroenterol 10 (2004) 1466-1470.

[27] A. Mulla, C. Leroux, E. Solito, J.C. Buckingham, Correlation between the antiinflammatory protein annexin 1 (lipocortin 1) and serum cortisol in subjects with normal and dysregulated adrenal function, J. Clin. Endocrinol. Metab 90 (2005) $557-562$.

[28] R. Paula, A.H. Oliani, D.C. Vaz-Oliani, S.C. D'Ávila, S.M. Oliani, C.D. Gil, The intricate role of mast cell proteases and the annexin A1-FPR1 system in abdominal wall endometriosis, J. Mol. Histol 46 (2015) 33-43.

[29] N. Chiang, C.N. Serhan, S.E. Dahlén, J.M. Drazen, D.W. Hay, G.E. Rovati, et al., The lipoxin receptor ALX: potent ligand-specific and stereoselective actions in vivo, Pharmacol. Rev. 58 (2006) 463-487.

[30] G. Leoni, P.A. Neumann, N. Kamaly, M. Quiros, H. Nishio, H.R. Jones, et al., Annexin A1-containing extracellular vesicles and polymeric nanoparticles promote epithelial wound repair, J. Clin. Invest 125 (2015) 1215-1227.

[31] P.G. Trentin, T.P. Ferreira, A.C. Arantes, B.T. Ciambarella, R.S. Cordeiro, R.J. Flower, et al., Annexin A1 mimetic peptide controls the inflammatory and fibrotic effects of silica particles in mice, Br. J. Pharmacol 172 (2015) 3058-3071.

[32] F.N. Gavins, S. Yona, A.M. Kamal, R.J. Flower, M. Perretti, Leukocyte antiadhesive actions of annexin 1: ALXR- and FPR-related anti-inflammatory mechanisms, Blood 101 (2003) 4140-4147.

[33] M. Perretti, S.J. Getting, E. Solito, P.M. Murphy, J.L. Gao, Involvement of the receptor for formylated peptides in the in vivo anti-migratory actions of annexin 1 and its mimetics, Am. J. Pathol 58 (2011) 1969-1973.

[34] A.P. Girol, K.K. Mimura, C.C. Drewes, S.M. Bolonheis, E. Solito, S.H. Farsky, et al., Anti-inflammatory mechanisms of the annexin A1 protein and its mimetic peptide Ac2-26 in models of ocular inflammation in vivo and in vitro, J. Immunol 190 (2013) 5689-5701.

[35] E. Ricciotti, G.A. FitzGerald, Prostaglandins and inflammation, Arterioscler Thromb. Vasc. Biol. 31 (2011) 986-1000.

[36] S. Seidel, H. Neymeyer, T. Kahl, T. Röschel, K. Mutig, R. Flower, et al., Annexin A1 modulates macula densa function by inhibiting cyclooxygenase 2, Am. J. Physiol. Renal. Physiol 303 (2012) 845-854.

[37] B.F. Barbosa, L. Paulesu, F. Ietta, N. Bechi, R. Romagnoli, A.O. Gomes, et al., Susceptibility to Toxoplasma gondii proliferation in BeWo human trophoblast cells is dose-dependent of macrophage migration inhibitory factor (MIF), via ERK1/2 phosphorylation and prostaglandin E2 production, Placenta 35 (2014) 152-162. 